Section Editor

Mitchell S.V. Elkind,

MD, MS

\title{
Clinical Reasoning: Encephalopathy in a 10-year-old boy
}

Lance Rodan, MD Ingrid Tein, MD, FRCPC

Correspondence \& reprint requests to Dr. Rodan: lance.rodan@utoronto.ca

\section{SECTION 1}

A 10-year-old, right-handed boy with a several-day history of fever and upper respiratory symptoms presented with acute onset headache, emesis, progressive mental status change, and right-sided focal seizures. Symptoms developed over approximately 3 hours.
There was no history of recent toxic or medication exposures, travel, immunizations, sick contacts, insect bites, or animal exposures.

The general review of systems was negative.

\section{Question for consideration:}

1. What is your initial differential diagnosis based on this information?

GO TO SECTION 2 


\section{SECTION 2}

Initial differential diagnosis should include infection (encephalitis or meningitis), inflammation (connective tissue disease/autoimmune disease, primary or secondary vasculitis, antineuronal antibody mediated encephalopathy), demyelination (e.g., acute disseminated encephalomyelitis), a vascular event (ischemic or hemorrhage), and a malignancy such as a glioma or lymphoma.

The patient was loaded with phenytoin and treated empirically with acyclovir and antibiotics while further history was obtained.

He was the product of a normal pregnancy and term delivery. His developmental history was normal.

Two years prior, he had a similar episode of fever and encephalopathy, which was associated with leftsided focal seizures and left hemiparesis. CT at that time demonstrated swelling of the right temporal lobe. He was presumptively diagnosed with herpes encephalitis, and received a full course of acyclovir. CSF herpes simplex virus (HSV) PCR was negative on 2 occasions. At his discharge from hospital, he had made a nearly complete recovery, with only mild residual left leg weakness.

Over the 2 years leading to his current admission, he continued to have persistent fatigue. Also, it became evident that he was having more difficulty in school than previously, and his grades dropped from As to Cs and Ds. In addition, when reviewing his growth curve, he had dropped several percentiles on his growth curve for both weight and height.
On family history, the mother has English and the father Hungarian heritage. Parents are nonconsanguineous. He has 2 younger twin male siblings who are healthy and developmentally normal. Family history is otherwise unremarkable.

On the current examination, he was mildly febrile and appeared pale. There was no meningismus. Glasgow Coma Scale (GCS) score was 13 due to confused, but fluent speech. He had a receptive aphasia. Pupils were equal and reactive to light and fundi were normal. He had a right superior quadrantanopia on visual threat. He had bilateral asymmetric ptosis. According to the parents, the ptosis had slowly developed over the last 2 years and was relatively constant throughout the day, but worsened when he was ill or fatigued. Smooth pursuit eye movements were normal. He had no facial weakness. Hearing was grossly normal bilaterally. Gag and jaw jerk were normal. On the motor examination, he had an asthenic build. He had bilateral pes cavus and hammertoes. Tone was decreased in the right arm and leg. Reflexes were $3+$ in the right arm and leg and $2+$ elsewhere. Plantar responses were upgoing bilaterally. The patient was spontaneously moving all 4 extremities, but had difficulty lifting his right arm and leg against gravity. According to his bedside nurse, his strength was increasing in the right side following his last seizure. He withdrew each of his 4 limbs to nailbed pressure.

\section{Question for consideration:}

1. Where is the lesion? 


\section{SECTION 3}

The patient likely has involvement of his left temporal lobe, including Wernicke area and inferior optic radiations. His right hemiparesis is possibly related to a postictal Todd paresis. His seizures could be spreading to his ipsilateral motor cortex from his temporal lesion, although a second lesion of the motor cortex cannot be excluded. His more chronic, bilateral ptosis with sparing of the pupils and extraocular movements could represent a rostral midbrain lesion affecting the central caudal nucleus, but more likely represents a neuromuscular process (neuromuscular transmission or myopathy). Finally, his pes cavus and hammertoes are possible evidence of a mild chronic polyneuropathy (although the differential diagnosis for these deformities also includes distal myopathy, very chronic myelopathy, inflammatory joint disorders, and familial pes cavus).

Question for consideration:

1. Does this information change your differential diagnosis? 


\section{SECTION 4}

Knowledge of a similar prior episode, and the additional history of longstanding constitutional symptoms, cognitive decline, chronic ptosis, and possible polyneuropathy brings a new dimension to the differential diagnosis.

A chronic vasculitis (primary or secondary) affecting the CNS and peripheral nervous system could be considered, but this would be unlikely since the patient does not have any other organ, joint, or skin involvement.

A paraneoplastic disease could also be considered, but these are relatively rare in children, with the exception of anti-NMDA encephalitis.

Mollaret meningitis or recurrent HSV encephalitis (e.g., from inherited Toll-like Receptor 3 mutations) could be considered. His school difficulties could be explained as the chronic sequelae of temporal lobe damage; however, there was never confirmation of HSV infection and this would not explain his peripheral nervous system involvement.

X-linked Charcot-Marie Tooth Disease (CMT1X) from mutations in connection 32 is rarely associated with transient encephalopathy and stroke-like episodes, but this would not account for the patient's systemic symptoms.

A chronic toxic exposure could be considered, but there is no history to support this.
Finally, an inborn error metabolism should be considered. The acute, recurrent presentation provoked by intercurrent illness suggests a small molecule disorder or disorder of energy metabolism. Involvement of both CNS and peripheral nervous system, and associated systemic symptoms, are common in mitochondrial disease. The history of 2 stroke-like episodes would be highly suggestive of mitochondrial encephalomyopathy, lactic acidosis, and stroke-like episode (MELAS) syndrome. Certain fatty acid oxidation (FAO) defects can present with episodic hypoketotic hypoglycemic encephalopathy, myopathy, exercise intolerance, and peripheral neuropathy (mitochondrial trifunctional protein deficiency and long-chain L-3-hydroxyl-acyl CoA dehydrogenase [LCHAD] deficiency), and patients can have permanent deficits if they have cerebral injury while hypoglycemic, though this tends to be generalized and not focal in distribution. Also, ptosis is not a typical feature for FAO disorders. Another potential metabolic etiology for recurrent strokes with headaches and cognitive decline is homocystinuria, though this is not associated with ptosis, neuropathy, exercise intolerance, or the described systemic involvement and is therefore unlikely.

\section{Question for consideration:}

1. What investigations would you order? 


\section{SECTION 5}

Complete blood count demonstrated a mild leukocytosis and normocytic anemia. Blood gas demonstrated a compensated metabolic acidosis. Initial lactate was $9.1 \mathrm{mmol} / \mathrm{L}$, and remained elevated on repeat samples. Pyruvate was not performed. Urine toxicology screen was negative.

CT head demonstrated a nonenhancing, hypodense mass lesion in the left temporal lobe and a small, chronic low density in the right parietal lobe. There was local mass effect, but no midline shift or effacement of quadrigeminal or suprasellar cisterns. Radiologic differential diagnosis included tumor, encephalitis, or infarct.

Lumbar puncture was performed and showed a normal cell count, normal glucose and protein, and a lactate of $5.29 \mathrm{mmol} / \mathrm{L}$ (upper limit of normal 2.4). CSF was sent for bacterial culture and viral PCR (HSV1/2, varicella zoster virus, Epstein-Barr virus, cytomegalovirus, HHV6, HHV7, HHV8, enterovirus, arbovirus including West Nile virus). Antimicrobials were discontinued when all cultures and viral studies returned as negative.

MRI was performed (figure) and showed a large, nonenhancing area of signal abnormality in the left temporal lobe with some mass effect and gyriform

\section{Figure MRI brain imaging in case patient}
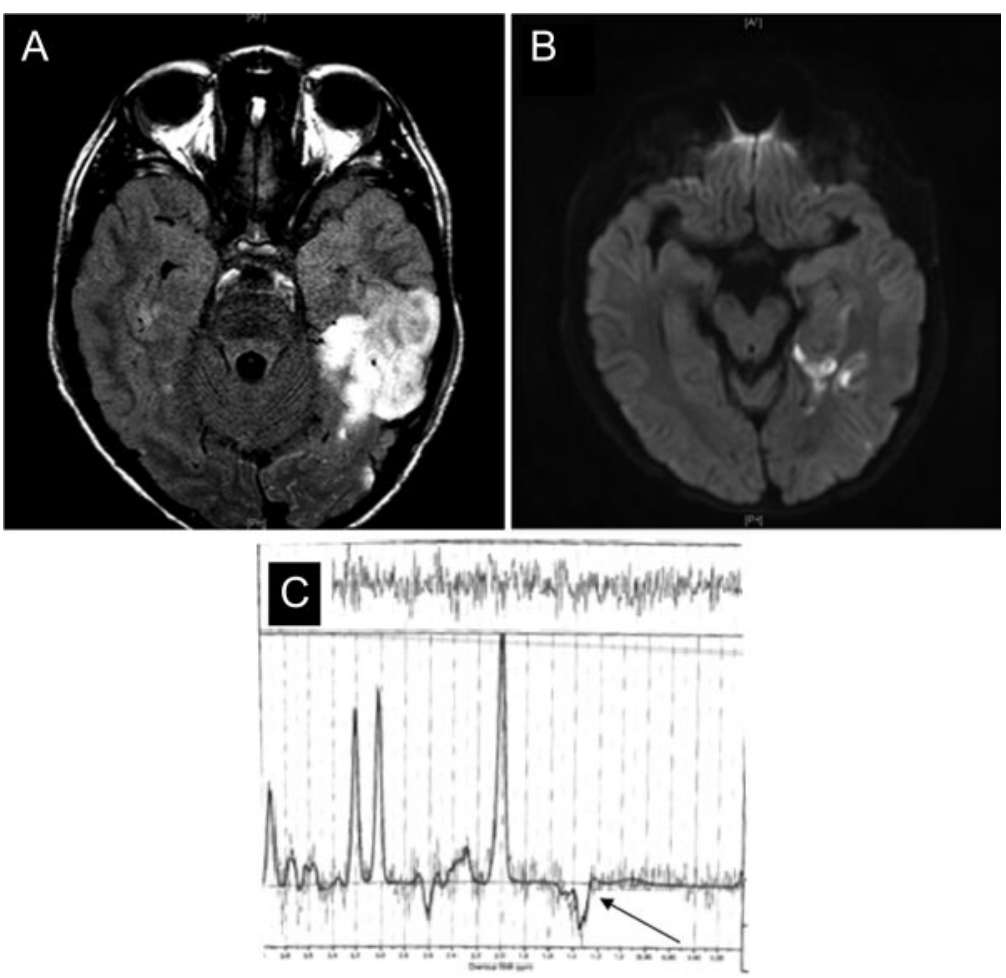

(A) Axial fluid-attenuated inversion recovery sequence demonstrates signal abnormality in the left temporal lobe with mass effect. (B) Axial diffusion-weighted sequence demonstrates gyriform cortical diffusion restriction. (C) Magnetic resonance spectroscopy with lactate peak at $1.33 \mathrm{ppm}$ (arrow). cortical diffusion restriction. There were also smaller, ill-defined areas of high fluid-attenuated inversion recovery signal of varying ages in the right superior temporal gyrus, right occipital lobe, left prefrontal gyrus, left superior temporal gyrus, and left postcentral gyrus. Magnetic resonance spectroscopy (MRS) showed a lactate peak at $1.33 \mathrm{ppm}$ (arrow).

Initial EEG was remarkable for slowing over the posterior aspect of the left hemisphere. There were no periodic lateralizing epileptiform discharges.

As a result of the clinical phenotype, genetic testing for mitochondrial DNA (mtDNA) $3243 \mathrm{~A} \rightarrow \mathrm{G}$ tRNA Leu and $3271 \mathrm{~T} \rightarrow \mathrm{C}$ tRNA Leu was sent. The patient was positive for the $3243 \mathrm{~A} \rightarrow \mathrm{G}$ tRNA Leu mutation with a mutation load of $32 \%$ in muscle.

DISCUSSION What is the diagnosis? MELAS refers to the syndrome of mitochondrial encephalomyopathy, lactic acidosis, and stroke-like episodes. The syndrome was first described by Pavlakis in 1984. The core features include 1) stroke-like episodes before the age of 40 years, 2) encephalopathy characterized by seizures, dementia, or both, and 3) lactic acidosis, ragged red fibers, or both, and supportive criteria included normal early development, recurrent headache, or recurrent vomiting. ${ }^{1}$ Onset of symptoms is frequently seen between the ages of 2 and 10 years. ${ }^{2}$ Stroke-like episodes refer to episodes of at least partially reversible neurologic deficits (often aphasia, hemianopia, and cortical blindness) that do not obey classic vascular territories. Posterior-parietal, temporal, and occipital cortices are preferentially involved, often asymmetrically. It is currently believed that the pathophysiology of these episodes includes both failure of oxidative metabolism at the cellular level in brain tissue itself as well as small vessel vasculopathy from mitochondrial failure in blood vessel endothelium and smooth muscle. ${ }^{3,4}$

While patients may recover from these stroke-like episodes, the disease follows a neurodegenerative course with accumulation of deficits over time. Migraine, sensorineural hearing loss, myopathy with exercise intolerance, and peripheral neuropathy are additional common neurologic features. Patients may also have involvement of systemic organs with a high oxidative demand, e.g., heart, gastrointestinal tract, pancreatic islets of Langerhans, and kidneys. Short stature is another common feature. ${ }^{5}$

The diagnosis of MELAS is based on a combination of clinical findings and molecular genetic testing. While most patients with the MELAS phenotype have the A3243G tRNA Leu mutation in their mitochondrial DNA, it is now known that the MELAS phenotype can result from many genetic defects, both in the mitochondrial and nuclear ge- 
nomes (e.g., complex 1 structural units encoded by mtDNA such as MT-ND5 and polymerase gamma [POLG] nuclear mutations). 5 The MELAS phenotype may also be part of an overlap syndrome with characteristics of other mitochondrial diseases (e.g., myoclonic epilepsy with ragged-red fibers [MERRF] syndrome, Leigh syndrome). ${ }^{5}$ All mitochondrial defects are maternally inherited, whereas nuclear defects usually demonstrate autosomal recessive inheritance. $^{5}$

Serum commonly demonstrates an elevated lactate with elevated lactate:pyruvate ratio, although lactate may be normal. Serum alanine (on quantitative amino acid analysis) may also be elevated. ${ }^{6}$

MRI often demonstrates signal change in the affected cortex, often sparing the subcortical white matter. The basal ganglia may also be involved. Diffusion-weighted imaging can show selective involvement of the cortical ribbon. MRS often reveals a characteristic lactate peak at $1.33 \mathrm{ppm}$, although this finding is not specific to mitochondrial disease and can be found in vascular stroke, hypoxic-ischemic injury, and infection. ${ }^{5,6}$

In MELAS associated with A3243G mitochondrial tRNA Leu mutations, pathology often demonstrates ragged red fibers on modified Gomori trichrome staining, representing the compensatory proliferation of abnormal subsarcolemmal mitochondria. Immunohistochemical staining may reveal variably decreased staining for complexes I and IV, while staining for the exclusively nuclear encoded complex II (succinate dehydrogenase) may be increased as a result of mitochondrial proliferation., In addition, there may be evidence of lipid accumulation. ${ }^{6}$ Electron microscopy may reveal proliferation of mitochondria, giant mitochondria, or mitochondrial inclusions. ${ }^{6}$ It should be stressed that a respiratory chain enzyme biochemistry panel should also be performed by a qualified laboratory on all muscle samples in patients suspected of a mitochondrial disease. The activity of complexes I to III, II to III, and IV are most commonly measured as a first line. The respiratory chain enzyme biochemistry may represent the only abnormality present in a child with a mitochondrial disease, and the pattern of abnormal complexes may suggest a particular molecular diagnosis. For a more detailed review of the in-depth investigation of suspected mitochondrial disease, the reader is referred to a recent review article. ${ }^{6}$

How would you manage this patient? In general, current management is aimed at slowing neurodegeneration and preventing stroke-like episodes, as well as acutely treating stroke-like episodes. Seizure control should be optimized, since breakthrough seizures may trigger stroke-like episodes. Valproate should be avoided if possible, as it is toxic to mitochondria, inhibits carnitine uptake in cells, and may exacerbate acute metabolic decompensation. ${ }^{7}$ Dichloroacetate may be used acutely to lower significant lactic acidosis but should not be used chronically because it may contribute to a severe peripheral neuropathy to which these individuals are already predisposed due to the mitochondrial disorder and any associated diabetes mellitus. ${ }^{8}$

Children with MELAS are often placed on a vitamin and antioxidant cofactor cocktail, variably including thiamine, riboflavin, creatine, vitamin C, vitamin E, $\alpha$-lipoic acid, coenzyme Q10/idebenone, and L-carnitine. There is limited prospective randomized double-blind control study evidence to support the use of any of these, but it is generally believed that there may be a theoretical benefit and little risk of harm in supplementing with these agents. ${ }^{9}$ The use of $\mathrm{L}$-arginine in the acute treatment of stroke-like episodes has been studied. IV doses of $500 \mathrm{mg} / \mathrm{kg}$ were given within 3 hours of the onset of symptoms. The arginine must be infused slowly over 15-30 minutes, monitoring for hypotension. In the subacute stage, the arginine can be continued orally at $150-300 \mathrm{mg} / \mathrm{kg} /$ day in 3 divided doses, provided there is normal renal function. Common side effects include nausea, vomiting, and abdominal pain. Small studies have shown efficacy for IV L-arginine used acutely in this manner. Furthermore, long-term treatment may decrease recurrence of stroke-like episodes. ${ }^{10}$ Larger prospective studies will be required to determine treatment efficacy.

\section{DISCLOSURE}

The authors report no disclosures relevant to the manuscript. Go to Neurology.org for full disclosures.

\section{REFERENCES}

1. Hirano M, Ricci E, Koenigsberger MR, et al. MELAS: an original case and clinical criteria for diagnosis. Neuromuscul Disord 1992;2:125-135.

2. DiMauro S, Hirano M. MELAS. In: GeneReviews. Available at: www.ncbi.nlm.nih.gov/books/NBK1233. Accessed October 24, 2011.

3. Gilchrist JM, Sikirica M, Stopa E, Shanske S. Adult onset MELAS: evidence for involvement of neurons as well as cerebral vasculature in strokelike episodes. Stroke 1996;27: 1420-1423.

4. Clark JM, Marks MP, Adalsteinsson E, et al. MELAS: clinical and pathologic correlations with MRI, xenon/CT, and MR spectroscopy. Neurology 1996;46:223-227.

5. Sproule DM, Kaufmann P. Mitochondrial encephalopathy, lactic acidosis, and strokelike episodes: basic concepts, clinical phenotypes, and therapeutic management of MELAS syndrome. Ann NY Acad Sci 2008;1142:133158.

6. Mitochondrial Medicine Society's Committee on Diagnosis. The in-depth evaluation of suspected mitochondrial disease. Mol Genet Metab 2008;94:16-37. 
7. Tein I, DiMauro S, Xie ZW, De Vivo DC. Valproic acid impairs carnitine uptake in cultured human skin fibroblasts: an in vitro model for the pathogenesis of valproic acid-associated carnitine deficiency. Pediatr Res 1993;34:281-287.

8. Kaufmann P, Engelstad K, Wei Y, et al. Dichloroacetate causes toxic neuropathy in MELAS: a randomized, controlled clinical trial. Neurology 2006;66:324-330.
9. Santa KM. Treatment options for mitochondrial myopathy, encephalopathy, lactic acidosis, and stroke like episodes (MELAS) syndrome. Pharmacotherapy 2010;30: 1179-1196.

10. Koga Y, Povalko N, Nishioka J, Katayama K, Kakimoto $\mathrm{N}$, Matsuishi T. MELAS and L-arginine therapy. Mitochondrion 2007;7:133-139. 


\title{
Neurology
}

\author{
Clinical Reasoning: Encephalopathy in a 10-year-old boy \\ Lance Rodan and Ingrid Tein \\ Neurology 2012;79; e12-e18 \\ DOI 10.1212/WNL.0b013e31825fdf51
}

This information is current as of July 16, 2012

\section{Updated Information \& Services}

\section{References}

Subspecialty Collections

Permissions \& Licensing

Reprints including high resolution figures, can be found at: http://n.neurology.org/content/79/3/e12.full

This article cites 9 articles, 3 of which you can access for free at: http://n.neurology.org/content/79/3/e12.full\#ref-list-1

This article, along with others on similar topics, appears in the following collection(s):

All Cerebrovascular disease/Stroke

http://n.neurology.org/cgi/collection/all_cerebrovascular_disease_strok e

All Pediatric

http://n.neurology.org/cgi/collection/all_pediatric

Clinical neurology examination

http://n.neurology.org/cgi/collection/clinical_neurology_examination Clinical neurology history

http://n.neurology.org/cgi/collection/clinical_neurology_history

Mitochondrial disorders; see Genetics/Mitochondrial disorders http://n.neurology.org/cgi/collection/mitochondrial_disorders_see_gene tics-mitochondrial_disorders

Information about reproducing this article in parts (figures,tables) or in its entirety can be found online at:

http://www.neurology.org/about/about_the_journal\#permissions

Information about ordering reprints can be found online:

http://n.neurology.org/subscribers/advertise

Neurology ${ }^{\circledR}$ is the official journal of the American Academy of Neurology. Published continuously since 1951, it is now a weekly with 48 issues per year. Copyright Copyright (? 2012 by AAN Enterprises, Inc.. All rights reserved. Print ISSN: 0028-3878. Online ISSN: 1526-632X.

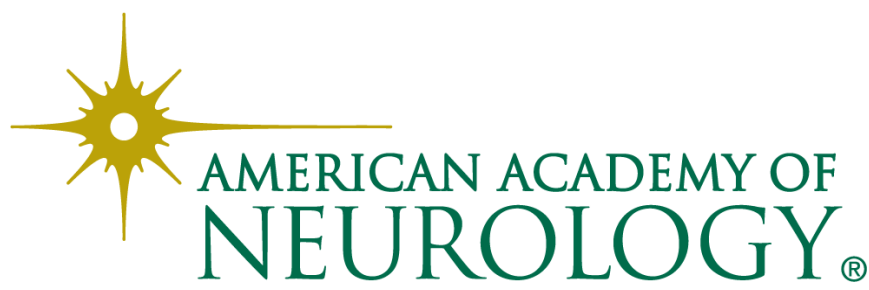

